\title{
Microestructura y polimorfismo en valvas recientes de Diplodon chilensis patagonicus (d'Orbigny 1835)
}

\author{
Microstructure and polymorphism Of Diplodon chilensis patagonicus (d'Orbigny 1835) \\ recent shells
}

\author{
Analía L. Soldati ${ }^{1,4^{*}}$, Dorrit E. Jacob ${ }^{1}$, María M. Bianchi ${ }^{2} \&$ Adán Hajduk ${ }^{3}$ \\ 1Departamento de Ciencias Geológicas, Universidad Johannes Gutenberg, Mainz, Becherweg 21, D-55099 Mainz, \\ Alemania. \\ ${ }^{2}$ Consejo Nacional de Investigacions Científicas y Técnicas, Centro Regional Universitario Bariloche, Universidad Nacional \\ del Comahue, Quintral 1250, CP8400 San Carlos de Bariloche, Río Negro, Argentina. \\ ${ }^{3}$ Consejo Nacional de Investigacions Científicas y Técnicas, Museo de la Patagonia, Centro Cívico, CP8400 San Carlos de \\ Bariloche, Río Negro, Argentina. \\ ${ }^{4}$ Consejo Nacional de Investigacions Científicas y Técnicas, Grupo de Caracterización de Materiales, Centro Atómico \\ Bariloche, Av. Bustillo 10500, San Carlos de Bariloche, Río Negro, Argentina \\ *Email: asoldati@cab.cnea.gov.ar
}

\section{RESUMEN}

Gracias a su potencial como registro ambiental, las valvas del molusco Diplodon han despertado recientemente el interés de la comunidad científica. En este trabajo, por medio de microscopía electrónica de barrido (SEM) y espectroscopía Raman se investigó la microestructura y el polimorfismo en valvas recientes de Diplodon chilensis patagonicus. Se encontró que la valva es completamente aragonítica y que la capa nacarada "externa" es la región más favorable para realizar mediciones de isótopos estables o elementos traza. Los análisis de microscopía confocal y SEM realizados demostraron que un incremento anual está compuesto de dos bandas: una de color claro, depositada durante los meses fríos y una de color oscuro, depositada durante los meses cálidos. Ambas bandas están formadas por nácar, compuesto de plaquetas poligonales de aproximadamente 5-7 $\mu \mathrm{m}$ de diámetro y 0.6-2 $\mu \mathrm{m}$ de espesor. Las plaquetas se encuentran ubicadas con el eje "c" del cristal (grupo cristalino: ortorrómbico) paralelo a la superficie de la valva y están unidas entre sí por medio de material orgánico inter-cristalino, traslúcido a los electrones. El nácar depositado sobre la marca anual de crecimiento muestra plaquetas de espesores más delgados, coincidiendo con la hipótesis de un crecimiento desacelerado asociado a la época de reproducción. Como consecuencia, en esta zona la proporción de material orgánico es mayor que la de $\mathrm{CaCO}_{3}$, y explica la formación de una línea azul más nítida, cuando la muestra es teñida con Alcian blue.

Palabras clave: Valva, Diplodon, microestructura, aragonita, líneas de crecimiento.

\begin{abstract}
Thanks to its potential as environmental archive, the valves of the freshwater clam Diplodon recently gained the attention of the scientific community. In this work, Scanning Electron Microscopy (SEM) and Raman Spectroscopy were used to investigate the material microstructure and polymorphism of recent Diplodon chilensis patagonicus shells. It was found that the shell is completely built of aragonite. In addition, the external nacreous layer shows to be the most appropriate to do stable isotopes and trace elements measurements. Analyses with confocal microscopy and SEM demonstrated that an annual increment is composed of two bands: one of lighter colors deposited during the cold months and one of darker colors deposited during the summer time. Both bands are nacre, composed of polygonal aragonite platelets of approximately 5-7 $\mu \mathrm{m}$ diameter and 0.6-2 $\mu \mathrm{m}$ thickness. The platelets have its "c"-axis (crystal group: orthorhombic) parallel to the shell surface and are bound together with an inter-crystalline organic material, translucent to the electrons. The nacre deposited in the annual growth mark shows thinner platelets, coinciding with the hypothesis of a decelerated growth associated to the reproduction time. As consequence, the valve presents there higher organic to $\mathrm{CaCO}_{3}$ ratios and explains why the annual mark is dyed more sharply when using Alcian blue.
\end{abstract}

KEYwoRDS: Shell, Diplodon, microstructure, aragonite, growth lines. 


\section{INTRODUCCIÓN}

Los moluscos bivalvos forman su valva por acreción periódica de carbonato de calcio $\left(\mathrm{CaCO}_{3}\right)$ y sustancias orgánicas desde el fluido extrapalial en el frente de la biomineralización (Addadi et al. 2006). Este fluido está situado entre el epitelio externo del manto y la superficie interna de la valva, y su composición está determinada tanto por la composición del agua que lo rodea, como por procesos metabólicos en el individuo (Crenshaw 1980). Bajo condiciones ambientales normales, este proceso está regulado por parámetros fisiológicos rítmicos, conocidos como "relojes biológicos" (Palmer 1970) y por eventos cíclicos externos como los ciclos de luz, temperatura o de mareas (Thompson 1975), que resultan en pronunciadas "líneas de crecimento" periódicas (diarias, con régimen de mareas, estacionales, anuales, etc.; Clark II 1974). A parte de estos ciclos y ritmos naturales, condiciones ambientales anormales tales como temperaturas extremas, tormentas, etc., también pueden provocar una alteración del crecimiento y formar "líneas de interrupción" (Clark II 1974). En muchos casos, estas líneas de crecimiento y de interrupción pueden ser útiles para crear una escala interna que sirva para datar exactamente el eje temporal en cada porción de la valva y ordenar en forma cronológica la información ecológica archivada en el molusco (Rhoads \& Panella 1970, Hudson et al. 1976).

La variación de parámetros ambientales, como por ejemplo el suplemento de nutrientes, el tipo de sustrato, la salinidad, la iluminación, la temperatura, y la concentración de oxígeno y dióxido de carbono disueltos, entre otros factores, pueden afectar el patrón de crecimiento, la estructura, la mineralogía, el fraccionamiento isotópico y la química de la valva. (Rhoads \& Panella 1970; Bertine \& Goldberg 1972; Meenakshy et al. 1974; Eisma et al. 1976; Carter 1980; Lutz \& Rhoads 1980; Brooks \& Rumsby 1984). Por ello las características de la valva, los patrones en la composición de elementos menores y trazas, y las señales isotópicas pueden servir como un archivo de la historia ambiental (por ejemplo, Schöne et al. 2007). Utilizando el patrón de crecimiento incremental como un calendario, la información química puede ser alineada cronológicamente en una escala anual, estacional, quincenal, diaria e incluso horaria (Clark II 1974), extendiendo vastamente el potencial del animal como un monitor de largo plazo del ecosistema acuático (para muestras recientes), e incluso como un archivo paleoecológico y paleoclimático (para muestras fósiles) (Rhoads \& Lutz 1980).

Mientras el potencial de los bivalvos para reconstrucciones ambientales está siendo cada vez más reconocido, la mayoría de los estudios se ha centrado en especies marinas (algunos ejemplos son los trabajos de Steuber 1999; Dutton et al. 2002, Schöne et al. 2002, Schöne et al. 2003, Steuber \& Rauch 2005, Pearce \& Mann 2006), mientras que los moluscos de agua dulce han sido menos investigados (por ejemplo: Nyström et al. 1995, Tevesz et al. 1996, Dettman et al. 1999, Wurster \& Patterson 2001, Dunca et al. 2005, Kaandorp et al. 2005, Carroll et al. 2006, Schöne B. et al. 2004, 2007). En Sudamérica, la almeja de agua dulce del género Diplodon (Bivalvia: Hyriidae) es uno de los bivalvos más abundantes a ambos lados de la Cordillera Andina, incluyendo las subespecies Diplodon chilensis chilensis (Gray, 1828) y Diplodon chilensis patagonicus (d'Orbigny, 1835) (Castellanos 1959a,1960); ésta última se encuentra en Argentina entre Mendoza (32 ${ }^{\circ} 52^{\prime} \mathrm{S} ; 68^{\circ}$ $\left.51^{\prime} \mathrm{W}\right)$ y Chubut $\left(45^{\circ} 51^{\prime} \mathrm{S} ; 67^{\circ} 28^{\prime} \mathrm{W}\right)$, e incluye el área de estudio de este trabajo: los lagos y ríos del Parque Nacional Nahuel Huapi (Castellanos 1959b, 1960, Semenas \& Brugni 2002). Gracias a sus bien estudiadas características de filtrador (Soto \& Mena 1999), los tejidos musculares, vísceras y contenidos digestivos del Diplodon han sido objeto de monitoreos de larga duración de la calidad del agua (Soto \& Mena 1999, Lara et al. 2002a) y de estudios de polución (Lara et al. 2002b, Ribeiro Guevara et al. 2004, 2005, Parada et al. 2008) en la región.

Recientemente, un estudio sistemático utilizando isótopos estables y elementos trazas combinado con análisis esclerocronológicos en ejemplares adultos de Diplodon chilensis patagonicus ha demostrado que este molusco desarrolla su valva en forma periódica e incremental (Soldati et al. 2009). En ese trabajo se reportó que la valva crece siguiendo una función sigmoidal, alcanzando incluso edades de hasta 90 años, con tasas de crecimiento promedio del orden de los $0.16 \mathrm{~mm} /$ año en la madurez. También se encontró que tanto los isótopos estables del oxígeno $\left(\delta^{18} \mathrm{O}\right)$ como ciertos elementos trazas $(\mathrm{Mn}, \mathrm{Ba}, \mathrm{Sr}$, $\mathrm{Mg}$ y S) se distribuyen en la valva del Diplodon siguiendo un comportamiento periódico, relacionado al esquema de crecimiento. Sin embargo, existen pruebas contundentes de que el fraccionamiento isotópico y la concentración de ciertos elementos traza dependen del hábito cristalino (tipo de microestructura) y del tipo de polimorfo del $\mathrm{CaCO}_{3}$ (Turekian et al. 1960, Tarutani et al. 1969, Rosenberg, 1980; Carriker et al. 1991, Melancon et al. 2005, Kim et al. 2007, Soldati et al. 2008). Por lo tanto, para poder desarrollar el potencial del Diplodon como biomonitor de condiciones ambientales pasadas y presentes en los ecosistemas andinos y subandinos, es necesario primero adquirir el conocimiento básico y detallado sobre la microestructura y la mineralogía del material estudiado. El presente trabajo, complementario a Soldati et al. (2009), es un estudio básico a nivel microscópico, mineralógico y estructural en valvas recientes de la subespecie Diplodon chilensis patagonicus. Con el mismo se pretende demostrar qué porción de la valva es más apta para realizar mediciones 
de elementos trazas e isótopos estables, así como también determinar qué elementos microestructurales conforman las líneas de crecimiento utilizadas para los estudios de esclerocronología.

\section{MATERIALES Y MÉTODOS}

Ejemplares de Diplodon chilensis patagonicus fueron colectados vivos en lagos y ríos del Parque Nacional Nahuel Huapi y alrededores, en marzo de 2007. Inmediatamente luego de la recolección, los moluscos fueron sacrificados y las partes blandas removidas. Las valvas fueron lavadas, limpiadas con un cepillo y dejadas secar al aire.

Para los estudios de las líneas de crecimiento se utilizaron las valvas izquierdas del molusco exclusivamente. Luego de cubrirlas con una resina metálica tipo epoxi bifenol-A (WIKO, Greussenheim, Alemania), las mismas fueron cortadas en forma perpendicular a las líneas de crecimiento, a lo largo del eje de altura con una sierra Isomet 1000 (Buehler, Illinois, USA) de baja velocidad. Las muestras fueron enrasadas en una superficie de vidrio con polvo de SiC de tamaño de grano 800, 1000 y 1200 , y el pulido final se hizo a $1 \mu \mathrm{m}$ con $\mathrm{Al}_{2} \mathrm{O}_{3}$ en un paño de pulido. Una vez alcanzado un pulido espejado, las muestras fueron inmersas en una tintura orgánica (Schöne et al. 2005) por 27 minutos a $38^{\circ} \mathrm{C}$ y secadas al aire. Este método disuelve ligeramente el carbonato de calcio con ácido acético, mientras que preserva la matriz orgánica con glutaraldehído y tiñe los azúcares y glicoproteínas del biomineral con Alcian blue. Las porciones de la valva con mayor proporción de material orgánico se tiñen de un color más intenso, facilitando así la identificación de las líneas de crecimiento (Soldati et al. 2009).

Fotografías digitales de las muestras fueron tomadas antes y después del tratamiento químico, con un microscopio binocular, bajo luz reflejada. También se utilizó un microscopio confocal con luz reflejada para fotografiar las muestras antes de la tinción.

Para el análisis de la microestructura, porciones de valvas fracturadas fueron cubiertas con una capa fina de grafito y analizadas utilizando un microscopio electrónico de barrido Leo 1530 acoplado con una columna de emisión GEMINI, en el Instituto Max Plank de Química de Mainz, división de Química de Partículas. Para la toma de imágenes se utilizaron un detector de electrones secundarios (SED) y uno de electrones de backscatter (BSD). Los parámetros usados fueron un voltaje de acelaración de 1-5 kV (75-80 $\mu \mathrm{A}$ ) y una distancia de trabajo de 2-3 $\mathrm{mm}$.

La mineralogía de los distintos componentes de las valvas se estudió por medio de espectroscopía Raman. Los espectros de las muestras pulidas (previas al teñido con la solución de Mutvei) fueron obtenidos a temperatura ambiente, utilizando un equipo Horiba Jobin Yvon LabRam HR con un detector CCD basado en Si y un microscopio óptico Olympus BX41 integrado. El mismo fue utilizado con los objetivos 10X, 50X y 100X. La radiación Rayleigh fue bloqueada con un filtro de canto y la luz reflejada fue dispersada con una red de 1800 grooves $/ \mathrm{mm}$. La línea de $532.2 \mathrm{~nm}$ del Láser $\mathrm{Nd}$ - YAG fue usada para la excitación, con $100 \mathrm{~mW}$ de potencia y un tamaño de muestreo de aproximadamente $2 \times 2 \mu \mathrm{m}$. Los espectros fueron adquiridos en ventanas de $400 \mathrm{~cm}^{-1}$ centradas en los picos de interés. El tiempo de adquisición varió entre 5 y $15 \mathrm{~s}$, y fue optimizado para lograr intensidades máximas sin alcanzar la saturación del detector. La adquisición de los datos, la restauración de la línea de base y el ajuste de los picos fueron realizados con el programa commercial LabSpec v4.02 (Jobin Yvon Horiba).

\section{RESULTADOS}

Las valvas de Diplodon chilensis patagonicus están compuestas de tres capas (Fig. 1a). La más externa es una capa orgánica color marrón llamada periostraco, y es la que está en contacto directo con el medio ambiente. El resto de la valva corresponde a una mezcla intrínseca de carbonato de calcio $\left(\mathrm{CaCO}_{3}\right)$ y sustancias orgánicas (proteínas y azúcares). La capa inmediatamente en contacto con el periostraco presenta una estructura prismática, color blanca, de un espesor aproximado de $100 \mu \mathrm{m}$. La capa siguiente posee una estructura nacarada, mide de 3 a $15 \mathrm{~mm}$ y presenta un patrón bien desarrollado de bandas claras y oscuras intercaladas (Fig. 1b). Esta última capa puede dividirse en dos partes, una subcapa externa (NE, Fig. 1b), en contacto íntimo con la capa prismática, y una subcapa interna (NI, Fig. 1b) en contacto íntimo con el epitelio del manto.

Los análisis por espectroscopía Raman demuestran que tanto la capa prismática como las nacaradas (interna y externa) tienen una estructura de aragonita (Fig. 2a). Este polimorfo es fácilmente reconocible en el espectro por un pico de gran intensidad en $1085 \mathrm{~cm}^{-1}$ correspondiente a la vibración simétrica elástica del ión carbonato y un doblete en 701-706 $\mathrm{cm}^{-1}$ debido a las vibraciones de la molécula en el plano (Fig. 2b).

Las imágenes tomadas por microscopía electrónica de barrido (Fig. 3) muestran que la capa nacarada de Diplodon chilensis patagonicus está compuesta de plaquetas poligonales irregulares (en general pentagonales o hexagonales) de aproximadamente 5-7 $\mu \mathrm{m}$ de diámetro y unos 0,6 a $2 \mu \mathrm{m}$ 
de ancho. La matriz orgánica, translúcida a los electrones, se observa en las microfotografías como una región oscura entre las plateletas de bio-aragonita. Comparando las plaquetas de aragonita ubicadas en la capa nacarada externa con las encontradas en la capa interna se observa que las plaquetas de la capa externa se encuentran en un excelente estado de conservación (Figs. 3a, 3b y 3c), mientras que las pertenecientes a la capa nacarada interna (Figs. 3d, 3e y 3f) se encuentran incompletas y parcialmente disueltas.

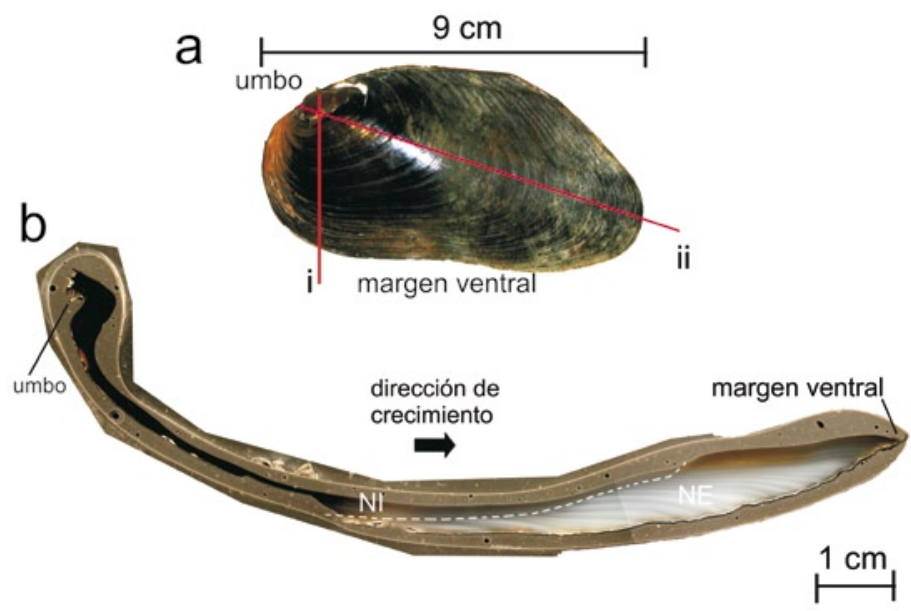

FiguRa 1. (a) Fotografía de la valva derecha en un ejemplar de Diplodon chilensis patagonicus indicando el eje de mínimo (I) y el de máximo (II) crecimiento. (b) Microfotografía de una sección transversal pulida (a lo largo del eje de mínimo crecimiento) mostrando la estructura de la valva. Contraste e iluminación fueron optimizados para resaltar las bandas claras y oscuras de la capa nacarada $(\mathrm{NI}=$ subcapa nacarada interna, $\mathrm{NE}=$ subcapa nacarada externa).

FIGURE 1. (a) Photography of the right valve of a Diplodon chilensis patagonicus specimen indicating the minimal (I) and maximal (II) growth axis. (b) Microphotography of a polished transversal section (following the minimal growth axis) showing the valve structure. Contrast and light were optimized to highlight the light/dark banding of the nacreous layer $(\mathrm{NI}=$ internal nacreous sub-layer, $\mathrm{NE}=$ external nacreous sub-layer).

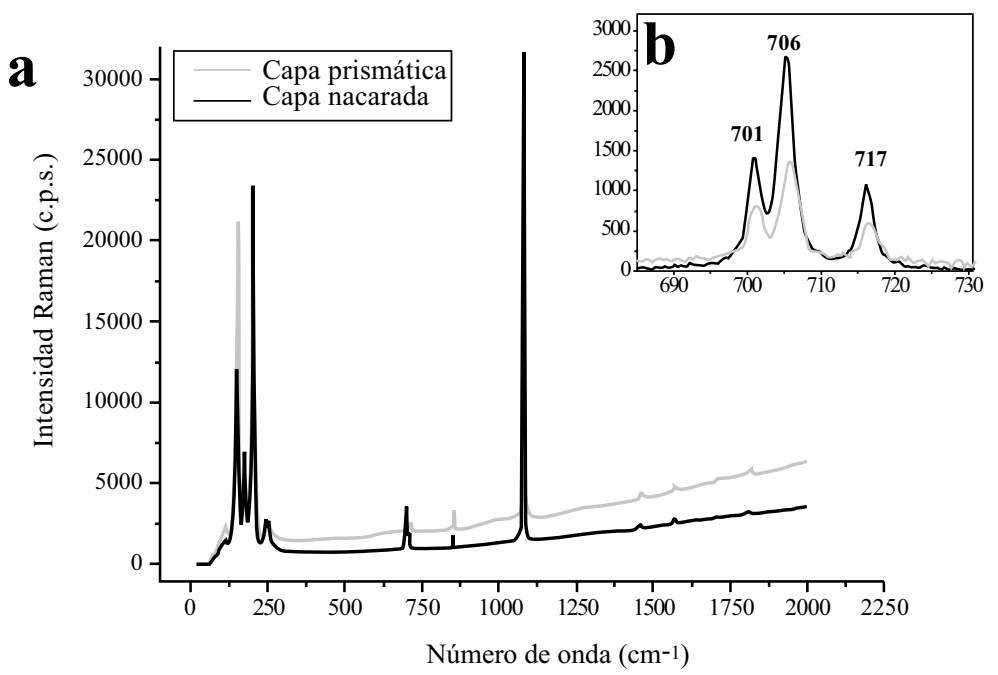

FIGURA 2. (a) Espectro Raman mostrando la mineralogía de la valva de Diplodon chilensis patagonicus, en la capa prismática y en la capa nacarada. El pico en $1085 \mathrm{~cm}^{-1}$ corresponde a la vibración elástica simétrica $\left(v_{1}\right)$ del ion carbonato $\left(\mathrm{CO}_{3}{ }^{2-}\right)$, las vibraciones en el plano $\left(v_{4}\right)$ producen una banda en la zona de los $700 \mathrm{~cm}^{-1}$, la región de 100-300 $\mathrm{cm}^{-1}$ corresponde a las vibraciones de la red. (b) Amplificación en la región de $700 \mathrm{~cm}^{-1}$ del espectro mostrado en 2a. El doblete en $701-706 \mathrm{~cm}^{-1}$ y el pico en $717 \mathrm{~cm}^{-1}$ son indicadores de la fase aragonita. En estas muestras no se observó calcita (reconocible por un pico en $\left.711 \mathrm{~cm}^{-1}\right)$. Abreviatura: c.p.s. = cuentas por segundo.

FIGURE 2. (a) Raman spectrum showing the mineralogy of the Diplodon chilensis patagonicus valve, in the prismatic and the nacreous layer. The band at $1085 \mathrm{~cm}^{-1}$ corresponds to the stretching symmetric vibration $\left(v_{1}\right)$ of the carbonate ion $\left(\mathrm{CO}_{3}^{2-}\right)$, the in-plane bending vibrations $(v)$ produce a band in the zone of $700 \mathrm{~cm}^{-1}$, the region between 100 and $300 \mathrm{~cm}^{-1}$ corresponds to the lattice modes vibrations. (b) Zoom at the region of $700 \mathrm{~cm}^{-1}$ in Figure 2a. The doublet in $701-706 \mathrm{~cm}^{-1}$ and the band in $717 \mathrm{~cm}^{-1}$ are indicating the presence of the polymorph aragonite. No calcite (band at $711 \mathrm{~cm}^{-1}$ ) could be identified in these samples. c.p.s. $=$ counts per second. 

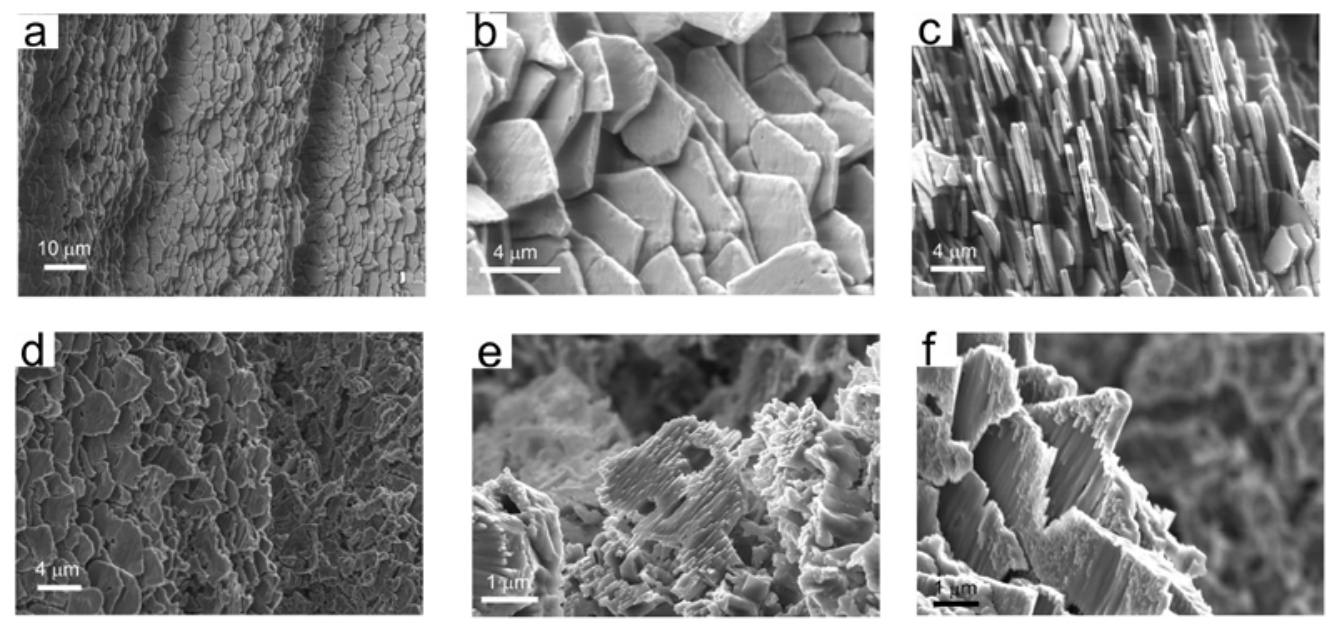

Figura 3. Imágenes de microscopio de barrido electrónico con columna de emisión de campo (FESEM) en valvas fracturadas de Diplodon chilensis patagonicus. (a) Capa nacarada externa (NE) de la valva. (b) plateletas de aragonita en NE vistas en forma paralela al eje c del cristal. (c) plateletas de aragonita en NE vistas en forma perpendicular al eje c del cristal. (d-f) capa nacarada interna (NI) semi disuelta con detalles de las plateletas.

Figure 3. Field Emission Scanning Electron Microscope (FESEM) micrographs of Diplodon chilensis patagonicus fractured valves. (a) External nacreous layer (NE) of the valve. (b) Aragonite polygons in NE showed parallel to the $\mathrm{c}$ axis of the crystal. (c) Aragonite polygons in NE showed perpendicular to the $\mathrm{c}$ axis of the crystal. (d-f) partially dissolved internal nacreous layer (NI) with details of the polygons.
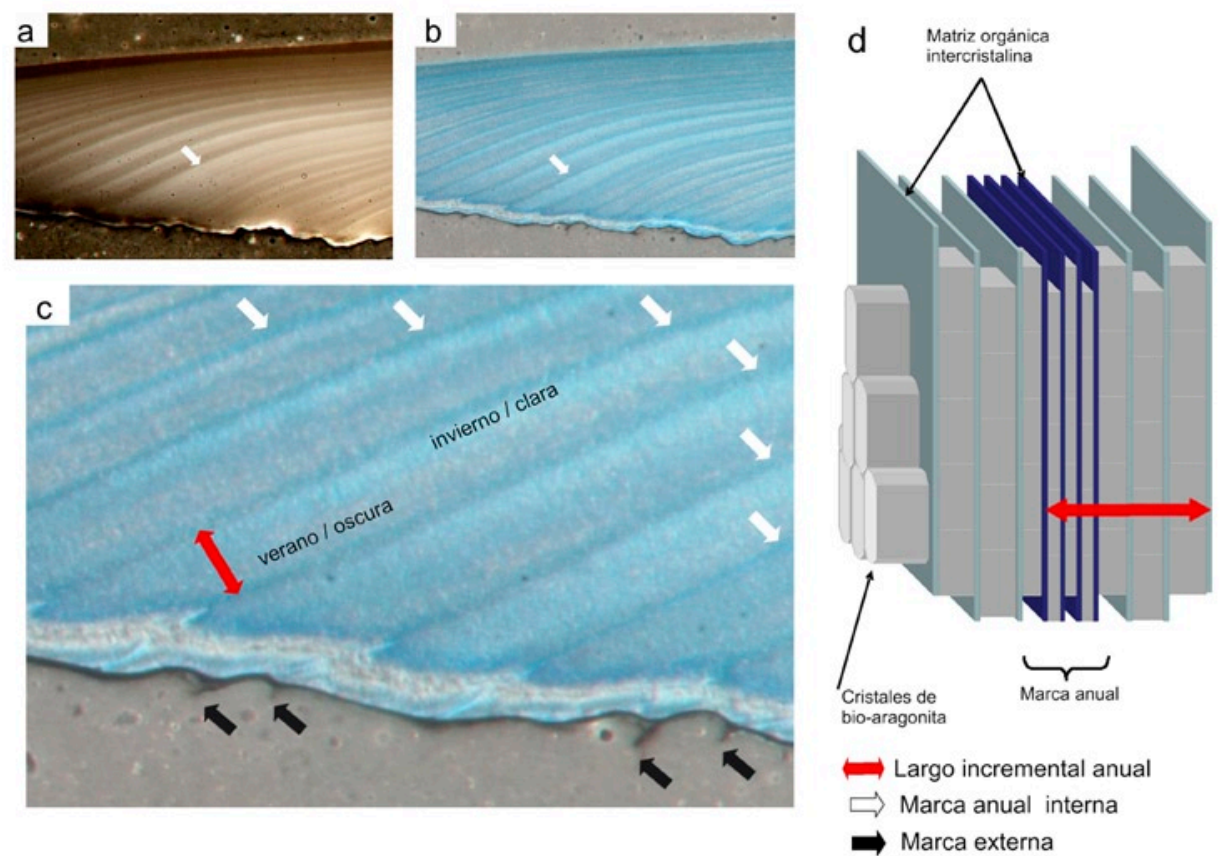

Figura 4. (a) Microfotografía de una sección pulida de Diplodon chilensis patagonicus antes y (b) después del tratamiento con Alcian blue. La flecha blanca marca el mismo punto en ambas fotografías. (c) Aumento de b mostrando las marcas anuales de interrupción de crecimiento, observadas como líneas azules nítidas; el material depositado en verano (líneas oscuras en a) se tiñe de un color azul más intenso que el material depositado durante el invierno (líneas claras de a). (d) esquema del crecimiento en las valvas, mostrando el largo incremental anual utilizado para los análisis esclerocronológicos.

Figure 4. (a) Microphotograph of a polished Diplodon chilensis patagonicus shell section before and (b) after the treatment with Alcian blue. The white arrow shows the same point in both pictures. (c) Zoom in b showing the annual growth marks, observed as narrow blue lines; the material deposited in summer (dark bands in a) is dyed with a more intense blue than the material deposited during the winters (light bands in a). (d) Sketch of the valve growth, showing the annual incremental length used for the sclerochronological analyses. 

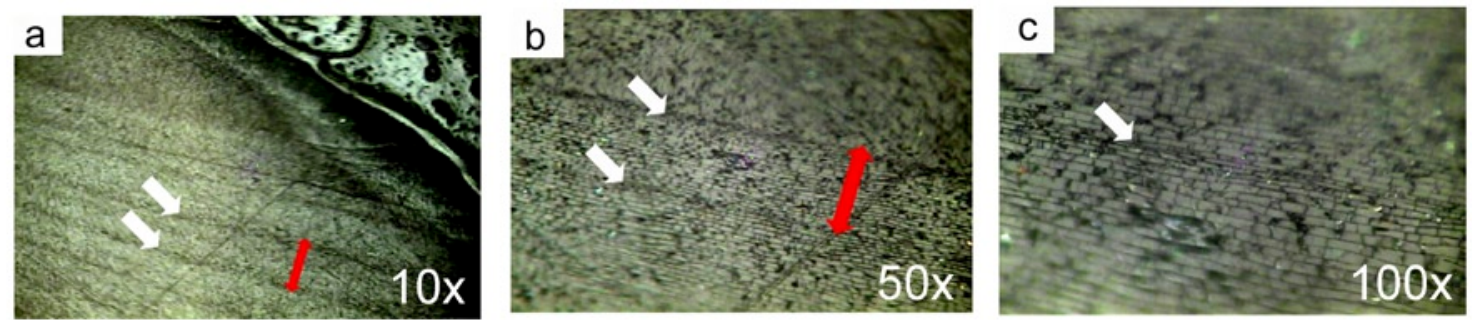

FIgURA 5. Microfotografías de una sección pulida de Diplodon chilensis patagonicus tomada con un microscopio confocal, colocando el foco $10 \mu \mathrm{m}$ por debajo de la superficie. Se muestra un incremento anual (flecha roja) y dos líneas de crecimiento consecutivas (flechas blancas). Amplificaciones usadas fueron de 10X (a), 50X (b) y 100X (c).

FIGURE 5. Microphotograph of a polished Diplodon chilensis patagonicus shell section with a confocal microscope; the focus was put 10 $\mu \mathrm{m}$ under the sample surface. An annual increment (red arrow) and two consecutive growth lines (white arrows) are shown. Amplifications used were 10X (a), 50X (b) and 100X (c).

Los incrementos periódicos en la capa nacarada externa del Diplodon chilensis patagonicus aparecen como bandas alternadas de líneas claras y oscuras (Fig. 4a). Luego del baño en la solución de Alcian blue (Fig. 4b), las líneas oscuras se tiñen de un color azul oscuro (Fig. 4c), mientras que las bandas claras toman un color azul claro (Fig. 4c), diferenciando efectivamente ambos incrementos. Este tratamiento también realza una línea nítida (flechas blancas en la Fig. 4c), ubicada entre la banda oscura y la clara (referida de aquí en adelante como "marca anual", ver discusión).

Comparando las microfotografías antes y después del proceso de teñido, se observa que la región de la marca anual no es una región únicamente orgánica. Por el contrario, la línea de crecimiento es una región de la valva donde las plaquetas de aragonita poseen un espesor más delgado que durante el resto del año (Fig. 5a-c). En forma esquemática (Fig. 4d), la región de la marca anual representa un período de crecimiento muy lento, en donde poco carbonato de calcio es depositado en la valva. En esta época, el ancho de las plateletas de bio-aragonita (Fig. 3) disminuye considerablemente, la proporción de materiales orgánicos respecto de minerales aumenta, y la zona se tiñe mas intensamente dando origen a la nítida línea azul que llamamos "marca anual" (Fig. 4c).

\section{DISCUSIÓN}

En un estudio de Diplodon chilensis chilensis (provenientes de diferentes lagos chilenos) se reportó que estas valvas están compuestas de tres subcapas: a) una nacarada, b) una prismática irregular, y c) una prismática irregular y homogénea (Valdovinos \& Pedreros 2007). Valiéndose de observaciones microscópicas los autores asociaron las bandas claro/oscuras a una estructura alternada nacarada/prismática. El presente estudio no respalda estas observaciones para la subespecie analizada en este trabajo. Por medio de microscopía electrónica de barrido (FE-SEM) y espectroscopía Raman se determinó aquí que las valvas de Diplodon chilensis patagonicus poseen, además del periostraco (orgánico), dos capas aragoníticas: una prismática (de sólo unos $100 \mu \mathrm{m}$ de ancho, ubicada inmediatamente debajo del periostraco) y una nacarada (de varios milímetros). Esta última puede dividirse en dos subcapas, una externa (NE) y una interna (NI). Las imágenes tomadas con ayuda del FE-SEM en muestras fracturadas demuestran que tanto las bandas claras como las oscuras de la valva de Diplodon chilensis patagonicus corresponden solamente a una estructura nacarada: polígonos de aproximadamente 5-7 $\mu \mathrm{m}$ de diámetro por 0.6 a $2 \mu \mathrm{m}$ de ancho, similares a los encontrados en otros moluscos nacarados (por ejemplo: Mutvei 1977, Addadi \& Weiner 1997, Jacob et al. 2008). En este estudio no se encontraron indicios de ninguna estructura prismática relacionada a las bandas oscuras de la valva. Así mismo, en las muestras aquí analizadas tampoco se observaron regiones con estructura de calcita o vaterita (Wehrmeister et al. 2009). La única diferencia microestructural entre las regiones de bandas claras y las oscuras es el tamaño de las plateletas de nácar (Fig. 4d). En el caso de las bandas oscuras, las plateletas son más delgadas en la zona cercana a la marca anual, coincidiendo con una etapa de crecimiento lento, mientras que en las regiones claras, las plateletas son más anchas.

Por otro lado, en el presente trabajo se encontró también que la capa nacarada interna (NI) se encuentra parcialmente disuelta. Esto no parece ser un efecto de la preparación de las muestras, ya que el resto de valva, en las mismas muestras, se encontró siempre en un excelente estado de conservación. Por el contrario, se sugiere aquí que la disolución del carbonato en NI podría ocurrir durante la respiración anaeróbica. Es conocido que cuando el 
molusco comienza una respiración anaeróbica, el animal disuelve y reprecipita $\mathrm{CaCO}_{3}$ para mantener el $\mathrm{pH}$ interno, formando una solución tapón (Crenshaw 1980). Ya que estos procesos de disolución y reprecipitación podrían alterar la composición química e isotópica del nácar interno, consideramos razonable evitar el muestreo dentro de esta área. La zona nacarada externa (no afectada por este proceso) es la única parte de la valva de Diplodon chilensis patagonicus apta para realizar las mediciones de esclerocronología y fraccionamiento de isótopos.

En relación a la estacionalidad del crecimiento, de esta investigación se desprende que los incrementos periódicos en la capa nacarada externa del Diplodon chilensis patagonicus aparecen como bandas alternadas de líneas claras y oscuras, correspondientes a las estaciones de baja y alta temperatura ambiente respectivamente (Parada et al. 1989; Soldati et al. 2009). El baño en la solución de Alcian blue permite diferenciar claramente ambos incrementos, y así determinar con mayor facilidad una depositación estival de una invernal. Este tratamiento realza además una línea nítida ubicada entre la banda de verano y la de invierno, la cual aparece sólo una vez por año, y por lo tanto puede ser usada para calibrar anualmente la escala temporal en la valva (Soldati et al. 2009).

Con respecto a la formación de una marca anual, estudios de dinámica y densidad de poblaciones, ciclo reproductivo y estado embrionario (Gluzman de Pascar 1973, Peredo \& Parada 1986, Parada et al. 1987, 1990, Lara \& Parada 1988, Semenas \& Brugni 2002) muestran que la reproducción en el género Diplodon es estacional, provocando posiblemente la detención del crecimiento durante la gametogénesis en los meses de primavera-verano (Parada et al. 1989). Estos resultados coinciden con los obtenidos por este estudio para la subespecie Diplodon chilensis patagonicus: luego del teñido, las valvas muestran zonas altamente coloreadas al final de cada banda clara (invierno) y previo a la siguiente banda oscura (verano). Esto se debe a que la proporción de material orgánico respecto del $\mathrm{CaCO}_{3}$ aumenta al disminuir el tamaño de las plateletas de aragonita, de acuerdo con la hipótesis de un crecimiento desacelerado durante el período de reproducción.

Respecto a la determinación de parámetros de crecimiento, Parada et al. (1989) obtuvieron el crecimiento individual y poblacional de Diplodon chilensis chilensis utilizando en forma conjunta métodos experimentales de captura, marcación y recaptura combinados con métodos teóricos (método de Waldorf). Aunque el uso de métodos teóricos permita predecir correctamente el crecimiento a edad madura a partir de la evaluación del crecimiento en individuos jóvenes, éste procedimiento posee aún algunas desventajas, más relacionadas a la parte experimental que a la teórica.
Ejemplo son el esfuerzo experimental y el costo que implica el proceso de marcación y recaptura (al menos se deben emprender dos muestreos, uno para la etapa de marcación y uno para la recaptura) y el tiempo requerido hasta poder obtener un resultado (que es en general de al menos un año entre ambas etapas). Recientemente, Valdovinos \& Pedreros (2007) han estudiado el crecimiento de los anillos internos en Diplodon chilensis chilensis empleando un método de tinción de la aragonita, y validaron la correspondencia entre los anillos de crecimiento internos y externos en ejemplares jóvenes. Sin embargo los anillos externos del periostraco en Diplodon chilensis patagonicus (flechas negras, Fig. 4c) coinciden con los anillos internos (flechas blancas, Fig. 4c) sólo cuando el ejemplar es muy jóven. En ejemplares mayores a 10 años de vida, los anillos externos presentan varias desventajas como puede verse claramente en la Fig. 4c: primero, no siempre representan líneas de crecimiento interanuales; segundo, las distancias incrementales medidas en el periostraco (entre dos flechas negras sucesivas) no se corresponden con las distancias interanuales medidas en el interior de la valva (entre dos flechas blancas sucesivas, Fig. 4c); y tercero, en las partes más maduras los anillos externos se solapan, y por lo tanto no permiten reconocer cuándo comienza un año y termina otro. El análisis esclerocronológico de los anillos de crecimiento internos (en vez de los externos) ofrece en este caso dos ventajas críticas para el estudio de bivalvos de gran longevidad: 1) todos los anillos de crecimiento, independientemente del estado de desarrollo del individuo, pueden ser visualizados en forma fidedigna con el teñido con Alcian blue y 2) la determinación de la tasa de crecimiento puede llevarse a cabo sobre el tiempo de vida completo del especimen y no está restricto a individuos jóvenes, los que poseen tasas de crecimiento altamente mayores al promedio correspondiente a la vida madura.

\section{AGRADECIMIENTOS}

Los autores le agradecen a la Prof. Liliana Semenas (Universidad Nacional del Comahue) por haber provisto bibliografía sobre Diplodon, a Joachim Huth del Instituto Max Plank de Química de Partículas por su asistencia con el FESEM, a Benita Witt y Lars Beierlein por la preparación de las muestras y a las autoridades del Parque Nacional Nahuel Huapi por haber permitido el muestreo. Se agradecen así mismo los aportes de Isabel Cruz, Esperanza Parada y un revisor anónimo, que han contribuido a mejorar el artículo. Este trabajo fue realizado gracias a una cooperación bilateral germano/argentina y fue financiado por el Ministerio de Ciencia y Tecnología de Argentina (MINCyT), la BMBF alemana, y el programa Geocycles Cluster de la Universidad de Mainz. Ésta es la publicación número 628 del cluster de excelencia Geocycles, de la Universidad Johannes Gutenberg de Mainz. 


\section{BIBLIOGRAFÍA}

ADDADI, L. \& S. WeINER. 1997. Biomineralization: A pavement of pearl. Nature 389: 912-914.

Addadi, L., D. Joester, F. Nudelman \& S. Weiner. 2006. Mollusk shell formation: A source of new concepts for understanding biomineralization processes. Chemical European Journal 12: 980-987.

Bertine, K.K. \& E.D. Goldberg.1972. Trace-Elements in Clams, Mussels, and Shrimp. Limnology and Oceanography 17 (6): 877-884.

Brooks, R.R. \& M.G. RumsBy. 1984. The Biogeochemistry of Trace-Element Uptake by Some New-Zealand Bivalves. Limnology and Oceanography 10 (4): 521-527.

Carriker, M.R., C.P. Swann, R.S. Prezant \& C.L. Counts. 1991. Chemical elements in the aragonitic and calcitic microstructural groups of shell of the oyster Crassostrea virginica: a proton probe study. Marine Biology 109: 287-297.

Carroll, M., C. RomaneK \& L. Paddock. 2006. The relationship between the hydrogen and oxygen isotopes of freshwater bivalve shells and their home streams. Chemical Geology 234 (3-4): 211-222.

Carter, J.G. 1980. Environmental and biological controls of bivalve shell mineralogy and microstructure. En: Skeletal growth of aquatic organisms: Biological records of environmental change (Eds. Rhoads, D.C. \& Lutz, R.A.), pp. 203-249. Plenum Press, New York and London.

Castellanos, Z.A. 1959a. Algunas consideraciones sobre el género Diplodon (moluscos-pelecípodos). Notas del Museo XIX Zoología: 182.

Castellanos, Z.A. 1959b. Las especies del género Diplodon en la Argentina. 1er Congreso Sudamericano Zoología, La Plata, Argentina.

Castellanos, Z.A. 1960. Almejas nacaríferas de la República Argentina. Género Diplodon. Secretaría Agricultura, Publicación Miscelánea 421: 1-40.

Clark II, G.R. 1974. Growth lines in invertebrate skeletons. Annual Review of Earth and Planetary Sciences 2: 77-99.

Crenshaw, M.A. 1980. Mechanisms of shell formation and dissolution. En: Skeletal growth of aquatic organisms: Biological records of environmental change (Eds. Rhoads, D.C. \& Lutz, R.A.), pp. 115-129. Plenum Press, New York and London.

Dettman, D.L., A.K. Reische \& K.C. Lohmann. 1999. Controls on the stable isotope composition of seasonal growth bands in aragonitic fresh-water bivalves (unionidae). Geochimica et Cosmochimica Acta 63 (7/8): 1049-1057.

DuncA, E., B.R. SchöNE \& H. Mutvei. 2005. Freshwater bivalves tell of past climates: But how clearly do shells from polluted rivers speak? Palaeogeography, Palaeoclimatology, Palaeoecology 228: 43-57.

Dutton, A.L., K.C. Lohmann \& W.J. Zinsmeister. 2002. Stable isotope and minor element proxies for Eocene climate of Seymour Island, Antarctica. Paleocenography 17 (2): 1-13.

Eisma, D., W.G. MоoK \& H.A. DAs. 1976. Shell Characteristics, Isotopic Composition and Trace-Element Contents of Some Euryhaline Mollusks as Indicators of Salinity. Palaeogeography Palaeoclimatology Palaeoecology 19 (1): 39-62.
Gluzmande Pascar, C. 1973. Preliminary note on hermaphroditism and embryonic stages in Diplodon variabilis. The Veliger 13 (3): 213-214.

Hudson, J.H., E.A. ShinN, R.B. Halley \& B. Lidz. 1976. Sclerochronology; a tool for interpreting past environments. Geology 4: 361-364.

Jacob, D.E., A.L. Soldati, R. Wirth, J. Huth, U. Wehrmeister \& W. Hofmeister. 2008. Nanostructure, composition and mechanisms of bivalve shell growth. Geochimica et Cosmochimica Acta 72 (22): 5401-5415.

KaAndorp, R.J.G., H.B. Vonhof, F.P. Wesselingh, L.R. Pittman, D. Kroon \& J.E.V. Hinte. 2005. Seasonal Amazonian rainfall variation in the Miocene Climate Optimum. Palaeogeography, Palaeoclimatology, Palaeoecology 221: $1-6$.

Kim, S.T., J.R. O'Neil, C. Hillaire-marcel \& A. Mucci. 2007. Oxygen isotope fractionation between synthetic aragonite and water: Influence of temperature and $\mathrm{Mg}^{2+}$ concentration. Geochimica et Cosmochimica Acta 71(19): 4704-4715.

Lara, G.P. \& E. Parada. 1988. Distribución espacial y densidad de Diplodon chilensis (Gray, 1828) en el Lago Villarica $\left(39^{\circ} 18^{\prime} \mathrm{S} ; 72^{\circ} 05^{\prime} \mathrm{W}\right)$. Boletín de la Sociedad Chilena de Biología de Concepción 59: 105-114.

Lara, G.P., E. Parada \& S. Peredo. 2002b. Alimentación y conducta alimentaria de la almeja de agua dulce Diplodon chilensis (Bivalvia: Hyriidae). Gayana 66 (2): 107-112.

Lara, G., A. Contreras \& F. Encina. 2002a. The freshwater mussel Diplodon chilensis (Bivalvia: Hyriidae) potential biofilter to diminish coliform levels of water wells. Laboratory experiment. Gayana 66 (2): 113-118.

Lutz, R.A. \& D.C. RHOADs. 1980. Growth patterns within the molluscan shell. An overview. En: Skeletal growth of aquatic organisms: Biological records of environmental change (Eds. Rhoads, D.C. \& Lutz, R.A.), pp. 203-249. Plenum Press, New York and London.

Meenakshy, V.R., G. Donnay, P.L. Blackwel \& K.M. Wilbur. 1974. Influence of Substrata on Calcification Patterns in Molluscan Shell. Calcified Tissue Research 15 (1): 31-44.

Melancon, S., B.J. Fryer, S.A. Ludsin, J.E. Gagnon \& Z. Yang. 2005. Effects of crystal structure on the uptake of metals by lake trout Salvelinus namaycush otoliths. Canadian Journal of Fisheries and Aquatic Sciences 62(11): 2609-2619.

Mutver, H. 1977. The Nacreous Layer in Mytilus, Nucula, and Unio (Bivalvia). Calcified Tissues Research 24: 11-18.

Nyström, J., U. Lindh, E. Dunca \& H. Mutvei. 1995. A study of $M$. margaritifera shells from the River Pauliströmsan, S. Sweden. Nuclear Instruments and Methods in Physics Research B 104: 612-618.

PALMER, J.D. 1970. Introduction to biological rhythms and clocks. En: The biological clock, two views (Ed. Palmer, J.D.), pp. 1-14. Academic Press, New York

Parada, E., S. Peredo \& C. Gallardo. 1987. Esfuerzo reproductivo en Diplodon chilensis (Gray, 1828) (Bivalvia, Hyriidae). Una proposición para su determinación $(+)$. Boletín de la Sociedad Chilena de Biología de Concepción 58: 121-126.

Parada, E., S. Peredo, G. Lara \& I. Valdebenito. 1989. Growth, age and life span of the freshwater mussel Diplodon chilensis chilensis (Gray, 1828). Archiv für Hydrobiologie 115 (4): 563-573.

Parada, E., S. Peredo \& C. Gallardo. 1990. Tácticas reproductivas 
y dinámica poblacional de Diplodon chilensis (Gray, 1828) (Bivalvia: Hyriidae). Revista Chilena de Historia Natural 63: 23-35.

Pearce, N.J.G. \& V.L. Mann. 2006. Trace metal variations in the shells of Ensis siliqua record pollution and environmental conditions in the sea to the west of mainland Britain. Marine Pollution Bulletin 52 (7): 739-755.

Peredo, S. \& E. Parada. 1986. Reproductive cycle in freshwater mussel Diplodon chilensis chilensis (Mollusca: Bivalvia). The Veliger 28 (4): 418-425.

Rhoads, D.C. \& G. Panella. 1970. The use of molluscan shell growth patterns in ecology and paleoecology. Lethaia 3 (2): 143-161.

RhoAds, D.C. \& R.A. Lutz. 1980. Skeletal growth of aquatic organisms: Biological records of environmental change. Plenum Press, New York and London.750 pp.

Ribeiro Guevara, S., D. Bubach, P. Vigliano, G. Lippolt \& M.A. ARRIBERE. 2004. Heavy metal and other trace elements in native mussel Diplodon chilensis from Northern Patagonia Lakes, Argentina. Biological Trace Elements Research 102 (1-3): 245-263.

Ribeiro Guevara, S., M.A. Arribere, D. Bubach, P. Vigliano, A. Rizzo, M. Alonso \& R. SÁnchez. 2005. Silver contamination on abiotic and biotic compartments of Nahuel Huapi National Park lakes, Patagonia, Argentina. Science of the Total Environment 336: 1-3.

RosenBerG, G.D. 1980. An ontogenetic approach to the environmental significance of bivalve shell chemistry. En: Skeletal growth of aquatic organisms: Biological records of environmental change (Eds. Rhoads, D.C. \& Lutz, R.A.), pp. 115-129. Plenum Press, New York and London.

Schöne, B., D.H. Goodwin, K.W. Flessa, D.L. Dettman \& P.D. RoOpNARINE. 2002. Sclerochronology and Growth of the Bivalve Mollusks Chione (Chionista) fluctifraga and $C$. (Chionista) cortezi in the Northern Gulf of California, Mexico. The Veliger 45 (1): 45-54.

Schöne, B., K. Tanabe, D.L. Dettman \& S. Sato. 2003. Environmental controls on shell growth rates and $\delta^{18} \mathrm{O}$ of the shallow-marine bivalve mollusk Phacosoma japonicum in Japan. Marine Biology 142 (3): 473-485.

Schöne, B., N.A. Page, D.L. Rodland, J. Fiebig, S. Baier, S.O. Helama \& W. Oschmann. 2007. ENSO-coupled precipitation records (1959-2004) based on shells of freshwater bivalve mollusks (Margaritifera falcata) from British Columbia. International Journal of Earth Sciences 96: 525-540.

Schöne, B.R., E. Dunca, H. Mutvei \& U. Norlund. 2004. A 217 year record of summer air temperature reconstructed from freshwater pearl mussels (M. margarifitera, Sweden). Quaternary Science Reviews 23: 1803-1816.

Schöne, B.R., E. Dunca, J. Fiebig \& M. Pfeiffer. 2005. Mutvei's solution: An ideal agent for resolving microgrowth structures of biogenic carbonates. Palaeogeography, Palaeoclimatology, Palaeoecology 228: 149-166.

Semenas L. \& N. Brungi. 2002. Características poblacionales y ciclo de vida de Diplodon chilensis (d'Orbigny, 1835) (Hyriidae, Bivalvia) en el lago Gutiérrez (Patagonia, Argentina). Ecología Austral 12: 29-40.

Soldati, A.L., D.E. Jacob, U. Wehrmeister \& W. Hofmeister. 2008 Structural characterization and chemical composition of aragonite and vaterite in freshwater cultured pearls. Mineralogical Magazin 72(2): 579-592.

Soldati, A.L., D.E. Jacob, B.R. Schöne, M.M. Bianchi \& A. HAJDUK. 2009. Seasonal periodicity of growth and composition in valves of Diplodon chilensis patagonicus (D'Orbigny, 1835). Journal of Molluscan Studies 75: 75-85.

Soto, D. \& G. Mena 1999. Filter feeding by the freshwater mussel, Diplodon chilensis, as a biocontrol of salmon farming eutrophication. Aquaculture 171: 65-81.

Steuber, T. 1999. Isotopic and chemical intra-shell variations in low-Mg calcite of rudist bivalves (MolluscaHippuritacea): disequilibrium fractionations and late Cretaceous seasonality. International Journal of Earth Sciences 88: 551-570.

Steuber, T. \& M. Rauch. 2005. Evolution of the $\mathrm{Mg} / \mathrm{Ca}$ ratio of Cretaceous seawater: Implications from the composition of biological low-Mg calcite. Marine Geology 217 (3-4): 199-213.

Tarutani, T., R.N. Clayton \& T.K. Mayeda. 1969. Effect of polymorphism and magnesium substitution on oxygen isotope fractionation between calcium carbonate and water. Geochimica et Cosmochimica Acta 33(8): 987-996.

Turekian, K. K. \& R. L. Armstrong. 1960. Magnesium, Strontium, and Barium Concentrations and Calcite-Aragonite Ratios of Some Recent Molluscan Shells. Journal of Marine Research 18(3): 133-151.

Tevesz, M.J.S., E. Barrera \& S.F. Schwelgien. 1996. Seasonal variation in oxygen isotopic composition of two freshwater bivalves: Sphaerium striatinum and Anodonta grandis. Journal of Great Lakes Research 22 (4): 906-916.

THOMPSON, I. 1975. Biological clocks and shell growth in bivalves. En: Growth rhythms and the history of the Earth's rotation: Interdisciplinary Winter Conference on Biological Clocks and Changes in the Earth's Rotation: Geophysical and Astronomical Consequences. (Eds. Rosenberg, G.D. \& Runcorn, S.), pp. 149-161. John Wiley \& Sons, London.

Valdovinos, C. \& P. Pedreros. 2007. Geographic variations in shell growth rates of the mussel Diplodon chilensis from temperate lakes of Chile: Implications fro biodiverstiy conservation. Limnologica 37: 63-75.

Wehrmeister, U., A.L. Soldati, D.E. Jacob, T. Häger \& W. HOFMEISTER. 2009. Raman spectroscopy of synthetic, geological and biological vaterite: a Raman spectroscopic study. Journal of Raman Spectroscopy DOI 10.1002/ jrs. 2438.

Wurster, C.M. \& W.P. Patterson. 2001. Seasonal variation in stable oxygen and carbon isotope values recovered from modern lacustrine freshwater mollusks: Paleoclimatological implications for sub-weekly temperature records. Journal of Paleolimnology 26 (2): 205-218.

Recibido: 21.08.09

Aceptado: 17.03 .10 